\title{
Avaliação in vitro da ação do óleo essencial de capim limão (Cymbopogon citratus) sobre o carrapato bovino Rhipicephalus (Boophilus) microplus
}

\author{
SANTOS, F.C.C.*; VOGEL, F.S.F. \\ Departamento de Medicina Veterinária Preventiva (DMVP), Laboratório de Doenças Parasitárias (LADOPAR), \\ Universidade Federal de Santa Maria, CEP: 97105-900, Santa Maria-Brasil *carlini@portoweb.com.br
}

\begin{abstract}
RESUMO: O uso indiscriminado de produtos químicos no controle do carrapato bovino constitui a principal causa do gradativo aumento do número de cepas resistentes deste parasita às bases disponíveis no mercado. A utilização de óleos essenciais e extratos vegetais é uma prática antiga no controle de carrapatos, porém só recentemente tem recebido a devida atenção dos pesquisadores. O objetivo deste experimento foi avaliar a eficácia in vitro do óleo de capim limão (Cymbopogon citratus) sobre fêmeas ingurgitadas de Rhipicephalus (Boophilus) microplus através do exame de biocarrapaticidograma. Foram testadas seis diluições do óleo de $C$. citratus $(1 ; 5$; 10; 25; 50 e 100\%) em uma população de carrapatos resistentes a amidínicos e piretróides sintéticos. A inibição de postura foi de $3 ; 23 ; 46 ; 66 ; 46$ e 46\%, a eclosão larval foi de $83 ; 58 ; 31$; $0 ; 38$ e $25 \%$ e a eficácia do tratamento foi de $32 ; 64 ; 83 ; 100 ; 88$ e $82 \%$, respectivamente. O óleo de $C$. citratus apresentou controle parcial do carrapato $R$. microplus in vitro, mesmo frente a populações resistentes a produtos químicos.
\end{abstract}

Palavras-chave: carrapaticida, Cymbopogon citratus, fitoterápicos, plantas medicinais, Rhipicephalus (Boophilus) microplus

\begin{abstract}
In vitro evaluation of the action of lemon grass (Cymbopogon citratus) essential oil on the cattle tick Rhipicephalus (Boophilus) microplus. The indiscriminate use of chemical products to control the cattle tick is the main cause of the gradual increase in the number of strains of this parasite that are resistant to the bases currently available in the market. The use of essential oils and plant extracts is an ancient practice for tick control; however, only recently has it received due attention by researchers. The aim of this experiment was to evaluate the in vitro efficacy of lemongrass (Cymbopogon citratus) essential oil on engorged females of Rhipicephalus (Boophilus) microplus through immersion test. Six concentrations of Cymbopogon citratus oil (1; $5 ; 10 ; 25 ; 50$ and $100 \%$ ) were tested against a tick population resistant to synthetic formamidines and pyrethroids. The inhibition of egg-laying was $3 ; 23 ; 46 ; 66 ; 46$ and $46 \%$, the hatching was 83 ; $58 ; 31 ; 0 ; 38$ and $25 \%$, and the treatment efficacy was $32 ; 64 ; 83 ; 100 ; 88$ and $82 \%$, respectively. $C$. citratus oil showed partial control of the tick $R$. microplus in vitro, even against populations resistant to chemical products.
\end{abstract}

Key words: acaricide, Cymbopogon citratus, phytotherapeutic medicines, medicinal plants, Rhipicephalus (Boophilus) microplus

\section{INTRODUÇÃO}

O carrapato Rhipicephalus (Boophilus) microplus é o ectoparasita de maior impacto econômico na pecuária brasileira. Os principais prejuízos causados por este parasita são relacionados à baixa conversão alimentar, perda de peso crônica, redução da produção de leite, redução da qualidade do couro, lesões na pele que favorecem desenvolvimento de míases, anemia, transmissão de patógenos tais como Babesia bigemina, B. bovis e
Anaplasma marginale. O controle deste ácaroé realizado a base de produtos químicos há muitos anos, o que tem causado a seleção de populações de carrapato com resistência aos acaricidas. Neste contexto, a análise de técnicas alternativas de controle é uma área promissora de pesquisas e investimentos e entre estas áreas destaca-se a fitoterapia. A utilização de extratos vegetais no controle do carrapato tem sido foco de pesquisas a nível mundial (Alvaréz et al., 2008).

Recebido para publicação em 12/05/2011

Aceito para publicação em 29/03/2012

Rev. Bras. PI. Med., Botucatu, v.14, n.4, p.712-716, 2012. 
Cymbopogon citratus (DC.) Stapf (Poaceae) é erva perene, ereta, de $60 \mathrm{~cm}$ a 2 metros de altura, possui folhas esverdeadas, aromáticas, estreitas, longas e paralelinérveas partindo da base. Neste fitoterápico são encontrados flavonóides, alcalóides e triterpenos que lhe conferem várias atividades como antibacteriano, antifúngico, inseticida, diurético, anticarcinogênico, hipotensivo e anti-inflamatório (Silva et al., 2005). É uma planta medicinal que possui em sua estrutura os princípios ativos citral, geraniol, metileugenol, mirceno, citronelal, ácido acético e ácido caproico. O óleo essencial é extraído das folhas que, quando jovens, são compostas na maior parte de citral, sendo que a este componente é atribuído a maioria das propriedades farmacológicas da planta (Silva et al., 2010). Os óleos essências de plantas do gênero Cymbopogon têm sido alvo de pesquisas devido à ação carrapaticida (Chagas et al., 2012), assim como a possibilidade do uso dentro de sistema orgânico de criação de bovinos.

O objetivo deste experimento foi avaliar a eficácia de diferentes concentrações do óleo essencial $C$. citratus sobre teleóginas de $R$. microplus em experimentos in vitro.

\section{MATERIAL E MÉTODO}

Os testes in vitro foram conduzidos no Laboratório de Doenças Parasitárias da Universidade Federal de Santa Maria, entre os meses de abril e junho de 2010. Foi coletado um total de 270 teleóginas de R. microplus, em bovinos naturalmente infestados. No laboratório, as mesmas foram lavadas, secas e separadas em 9 grupos, com 10 teleóginas cada, baseado na técnica descrita por Drummond et al. (1973). O óleo essencial foi fornecido pela empresa Tecpon (Cachoerinha, Rio Grande do Sul, Brasil), tendo sido extraído da planta através do método de arraste de vapor.

O estudo foi constituído de 9 tratamentos, sendo que o grupo I correspondeu ao grupo controle não tratado, e os grupos II ao VII corresponderam às diferentes concentrações do óleo de $C$. citratus $(1 ; 5$; $10 ; 25 ; 50$ e $100 \%$, respectivamente). Os tratamentos VIII e IX corresponderam aos produtos químicos amitraz $12,5 \%$ e cipermetrina $5 \%$.

As teleóginas foram imersas em $20 \mathrm{~mL}$ das soluções correspondentes a cada tratamento por 5 minutos. Após a imersão, as teleóginas foram secas em papel toalha e fixadas através de fita adesiva em placas de Petri, previamente identificadas. A seguir, estas foram levadas à estufa climatizada $\left(27^{\circ} \mathrm{C}\right.$, UR $>80 \%$ ) por 14 dias. Após o período de postura, o total de ovos foi pesado e transferiu-se $0,3 \mathrm{~g}$ desse conteúdo para tubos de ensaio, vedados com algodão, sendo, novamente, encaminhadas para a estufa onde foram mantidas por 26 dias. Após o período de incubação dos ovos realizou-se a estimativa do percentual de eclodibilidade larval e, em seguida, foram calculadas a eficiência reprodutiva (ER) e a eficácia do tratamento (ET) de acordo com Drummond et al. (1973), através das seguintes fórmulas:

$$
\begin{aligned}
& E R=\underset{\text { peso dos ovos }}{\text { peso das teleóginas }} \% \text { de eclosão } \times 20.000 \\
& \text { peso }
\end{aligned}
$$

$$
\mathrm{ET}(\%)=\frac{(\mathrm{ER} \text { controle }-\mathrm{ER} \text { tratado })}{\mathrm{ER} \text { controle }} \times 100
$$

Os tratamentos foram realizados em triplicata e os resultados foram obtidos através das médias. A avaliação estatística foi realizada pelo método de análise de regressão através do programa SAS 9.0 Analyst, a um nível de significância de $5 \%$.

\section{RESULTADOE DISCUSSÃO}

Os tratamentos de I a VII apresentaram inibição de postura (IP) de 0; 3; 23; 46; 66; 46 e 46\% respectivamente. A eclosão larval (EL) de 100, 83; 58; 31; 0; 38 e 25\%, respectivamente. E a eficácia do tratamento (ET) de $0 ; 32 ; 64 ; 83 ; 100 ; 88$ e $92 \%$ respectivamente (Tabela 1). A IP, EL e ET para os produtos amitraz e cipermetrina foi de $40 ; 50 ; 71 \%$ e 40; $20 ; 90 \%$, respectivamente, sendo estes os tratamentos VIII e IX, respectivamente.

$\mathrm{Na}$ análise de regressão linear com os dados de inibição de postura $(x)$ e concentração do óleo de capim limão $(y)$ foi verificado associação não significativa $(p>0,05)$ expressa na equação $y=28,1$ $+0,356 x$. Com os dados de eclosão de postura $(x) e$ concentração do óleo $(x)$ foi verificada associação não significativa ( $p>0,05)$ expressa na equação $y=50,2$ $0,338 x$. Com os dados de eficácia do tratamento $(x)$ e concentração de óleo $(y)$ foi verificado associação significativa $(p<0,05)$ expressa na equação $y=64,7$ $+0,371 \times$ (Figura 1). Estes resultados indicam que 0 óleo de capim limão a $5 \%$ seria suficiente para se obter uma eficácia de $100 \%$ em avaliações in vitro e que concentrações superiores a $5 \%$ não necessariamente obtém eficácia máxima.

Para um produto químico ser considerado de alta eficácia ele precisa eliminar mais que $95 \%$ dos carrapatos, de média eficácia entre $90-95 \%$ e de baixa eficácia menos de 90\% (Brasil, 1990). Diante dos resultados dos produtos químicos testados podese afimar que esta população é resistente a compostos amidínicos e piretróides sintéticos. O óleo essencial de $C$. citratus a $25 \%$ ocasionou morte de todas as teleóginas, obtendo $100 \%$ de eficácia. Já os óleos a 50 e $100 \%$, apesar de estarem em maior concentração, apresentaram resultados inferiores, porém ainda relevantes na área da fitoterapia devido ao controle parcial dos carrapatos. Uma possível explicação seria 
TABELA 1. Médias percentuais de inibição de postura (IP), eclosão larval (EL) e eficácia do tratamento (ET) conforme as diferentes concentrações do óleo de Cympopogon citratus (C) sobre teleóginas de Rhipicephalus (Boophilus) microplus. Santa Maria, RS, Brasil, 2010.

\begin{tabular}{c|c|c|c|c}
\hline Tratamento & C (\%) & IP (\%) & EL (\%) & ET (\%) \\
\hline I & 0 & 0 & 100 & 0 \\
II & 1 & 3 & 83 & 32 \\
III & 5 & 23 & 58 & 64 \\
N & 10 & 46 & 31 & 83 \\
V & 25 & 66 & 0 & 100 \\
V & 50 & 46 & 38 & 88 \\
VII & 100 & 46 & 25 & 92 \\
VIII & amitraz & 40 & 50 & 71 \\
IX & cipermetrina & 40 & 20 & 90 \\
\hline
\end{tabular}

que o óleo puro tem densidade e capilaridade em níveis diferentes quando comparado ao diluído, e, por isto, o princípio ativo não apresenta o mesmo poder de penetração nos carrapatos formando uma película oleosa ao redor da teleógina. O óleo em alta concentração também apresenta inferior biodisponibilidade e solubilidade (Chagas et al., 2012). Os resultados indicam que esta planta apresentou controle parcial dos carrapatos e por isto merece maior atenção e pesquisas mais aprofundadas acerca da ação carrapaticida.

C. citratus já possui ação fitoterápica comprovada como antimicrobiano (Onawunmi et al., 1984) e contra nematoides gastrintestinais de caprinos em experimentos in vitro (Almeida et al., 2003).
Chungsamarnyart \& Jiwajinda (1992) estudaram destilados das folhas de $C$. citratus e de Cymbopogon nardus, observando que ambos são capazes de causar a morte de fêmeas ingurgitadas de $R$. microplus in vivo. Os resultados in vitro corroboram com os achados in vivo deste autores, afirmando uma ação carrapaticida da planta.

Broglio-Micheletti et al. (2009) testaram diversos extratos de fitoterápicos utilizados empiricamente no controle do carrapato bovino. Em ensaios in vitro, estes autores verificaram que após a imersão das teleóginas no extrato de $C$. citratus apenas a metade sobreviveu, no entanto, $75 \%$ dos ovos eclodiram e geraram larvas, sendo a eficácia do tratamento de $18 \%$. Silva et al. (2007) testando extrato

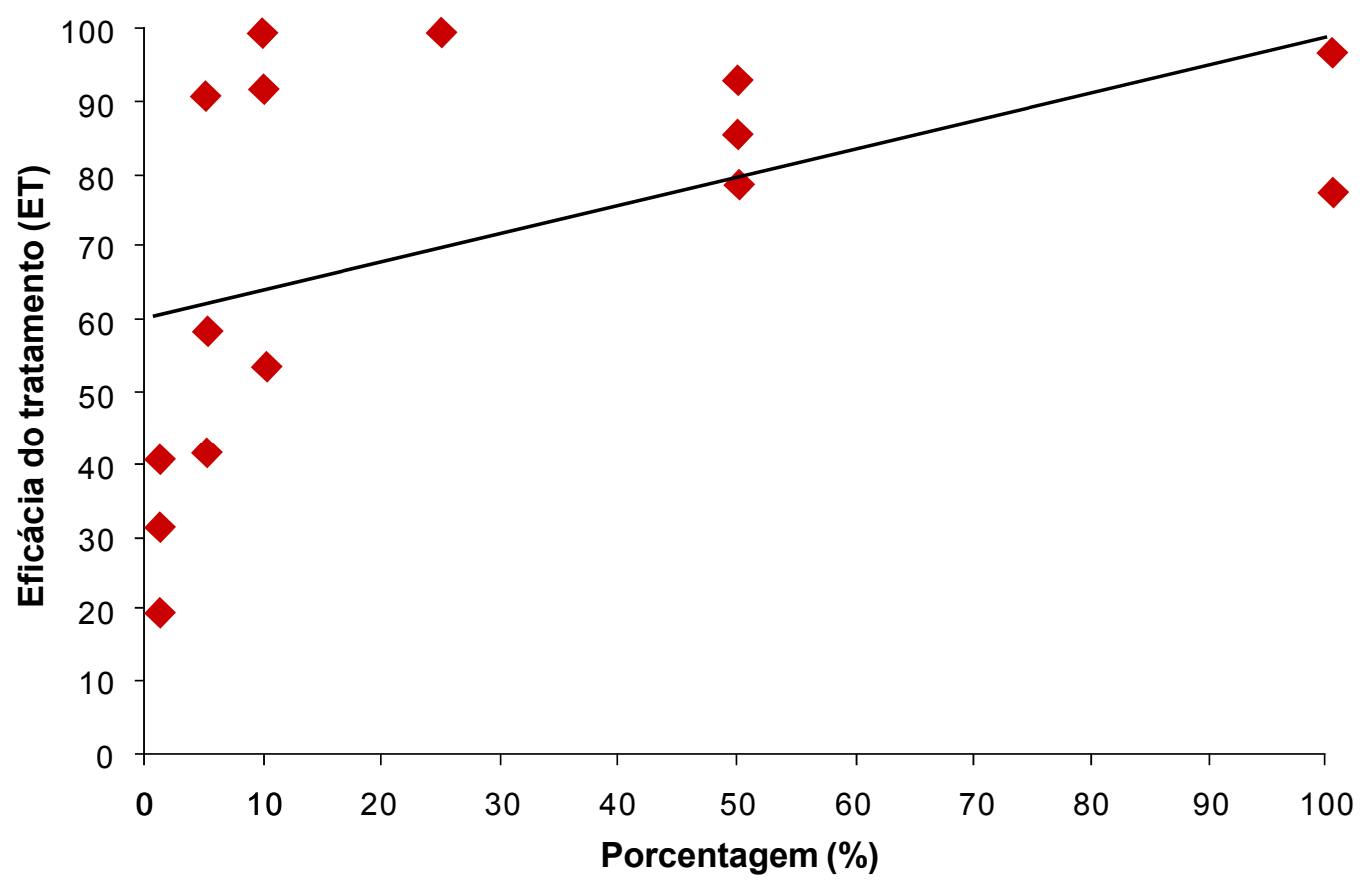

FIGURA 1. Análise de regressão linear com os parâmetros de eficácia do tratamento $(E T)(x)$ e concentração do óleo de capim limão (\%) (y) em teleóginas de R. microplus. Santa Maria, RS, Brasil, 2010. 
alcoólico de C. citratus, através do exame biocarrapaticidograma encontraram índice médio de eficácia de $42 \%$ e atribuíram este fato à capacidade desta planta em interferir na ovoposição e fecundação das fêmeas ingurgitadas. $O$ atual experimento apresenta resultados que corroboram com esta constatação. Silva et al. (2008) realizaram ensaios in vitro com a planta na forma de extrato bruto, solução aquosa, solução alcoólica a $50 \%$ e constataram baixa taxa de mortalidade do parasita, alta taxa de eclosão dos ovos, e que nenhum tratamento teve eficácia acima de $50 \%$. Os autores demonstraram que apesar da baixa eficácia, este fitoterápico apresenta controle parcial sobre o carrapato e sugerem pesquisas mais detalhadas sobre métodos de fracionamento dos extratos, identificação, purificação e caracterização estrutural das substâncias presentes no mesmo.

Carneiro et al. (2000) encontraram $68 \%$ de eficácia média in vitro testando óleo essencial de $C$. citratus em teleóginas de $R$. microplus, resultado similar ao deste experimento onde foi constatado $76,5 \%$ de média. Soares (2003), também in vitro, testando extrato bruto de $C$. citratus obteve eficácia máxima de $48 \%$, sendo que isto foi atribuído à formulação testada, uma vez que o veículo oleoso apresenta uma melhor difusão dos princípios ativos contidos nesta planta.

Heimerdinger et al. (2006) testaram o extrato alcoólico de $C$. citratus a $2,72 \%$ in vivo e encontraram eficácia média de 40; 46 e 41\% para os dias 3,7 e 14 pós tratamento, respectivamente. Em estudos in vitro, Heimerdinger et al. (2009) encontraram eficácia média de $45 \%$ utilizando o extrato alcoólico a $23 \%$, indicando controle parcial deste fitoterápico sobre o carrapato bovino. Corroborando com estes resultados, no presente experimento a eficácia de $1 \%$ em ensaios in vitro foi similar ao encontrado in vivo pelo autor citado, mesmo com diferente formulação do fitoterápico.

Clemente et al. (2006) em estudo in vitro com óleo essencial de $C$. citratus nas concentrações de $50 ; 25 ; 12,5$ e $6,25 \%$ obtiveram eficácia de 15; $10 ; 6$ e $1 \%$, respectivamente. Estes resultados diferem dos encontrados no presente estudo, onde a eficácia mínima foi de $32 \%$ e a maioria dos tratamentos foi superior a $80 \%$. Esta divergência pode ser atribuída ao diferente diluente utilizado pelos autores e a interação com os componentes do fitoterápico e poder de penetração nas teleóginas.

Uma possível explicação para divergência de resultados entre os autores seria a diferença quanto à forma de armazenamento e extração do óleo, a quantidade e a parte utilizada da planta, a época de colheita, o manejo do solo, entre outros. Com o intuito de prevenir esta situação seria necessária uma padronização no método de colheita, manipulação e extração do óleo essencial de C. citratus, assim como estudos fitoquímicos aprofundados sobre a sua composição e modo de ação. A escassa informação técnica a respeito da interação de resultados in vitro e in vivo também é limitação da fitoterapia.

\section{CONCLUSÃO}

O óleo de C. citratus apresenta controle parcial em teleóginas ingurgitadas de $R$. microplus em ensaios in vitro, e, após avaliações in vivo, pode possuir um uso promissor como carrapaticida, inclusive em populações resistentes a bases químicas.

\section{REFERÊNCIA}

ALMEIDA, M.A. et al. Efeitos dos extratos aquosos de folhas de Cymbopogon citratus (DC.) Stapf (capim santo) e de Digitaria insularis (L.) Fedde (capim açu) sobre cultivos de larvas de nematóides gastrintestinais de caprinos. Revista Brasileira de Parasitologia Veterinária, v.12, n.3, p.125-9, 2003.

ÁLVAREZ, V. et al. Control in vitro de garrapatas (Boophilus microplus; Acari: Ixodidae) mediante extractos vegetales. Revista de Biologia Tropical, v.56, n.1, p.291302, 2008.

BRASIL. Ministério da Agricultura. Portaria n. 90, de 04 dez. Normas para produção, controle e utilização de produtos antiparasitários. Diário Oficial, 22 jan, s. 1, col. 2, 1990.

BROGLIO-MICHELETTI, S.M.F. et al. Extratos de plantas no controle de Rhipicephalus (Boophilus) microplus (Canestrini, 1887) (Acari: Ixodidae) em laboratório. Revista Brasileira de Parasitologia Veterinária, v.18, n.4, p.44-8, 2009.

CARNEIRO, M.C.B. et al. Susceptibilidade in vitro de fêmeas ingurgitadas de $B$. microplus a alguns óleos vegetais. In: SIMPÓSIO DE PLANTAS MEDICINAIS DO BRASIL, 16., 2000, Recife, Anais..., Recife, 2000, 264p. CLEMENTE, M.A.S. et al. Avaliação do potencial acaricida de Cymbopogon nardus e Cymbopogon citratus no controle de fëmeas de Boophilus microplus (Acari: Ixodidae). In: SEMANA DE BIOLOGIA E MOSTRA DE PRODUÇÃO CIENTÍFICA, 29. e 12., 2006, Juiz de Fora, Anais... Juiz de Fora, 2006, 256p.

CHAGAS, A.C.S. et al. In vitro efficacy of plant extracts and synthesized substances on Rhipicephalus (Boophilus) Microplus (Acari: Ixodidae). Parasitology Research, v.110, n.1, p.295-303, 2012.

CHUNGSAMARNYART, N.; JIWAJINDA, S. Acaricidal activity of volatile oil from lemon and citronella grasses on tropical cattle ticks. Kasetsart Journal, National Science, v.26, p.46-51, 1992.

DRUMMOND, R.O. et al. Boophilus annulatus and Boophilus microplus: laboratory tests for insecticides. Journal of Economic Entomology, v.66, n.1, p.130-3, 1973. HEIMERDINGER, A. et al. Extrato alcoólico de capimcidreira (Cymbopogon citratus) no controle do carrapato (Boophilus microplus) de bovinos leiteiros. Revista Brasileira de Parasitologia Veterinária, v.15, n.1, p.379, 2006. 
HEIMERDINGER, A. et al. Extratos de capim-cidreira e amitraz em teste in vitro sobre o carrapato bovino. Livestock Research for Rural Development, v.21, n.1, article 5, 2009. Disponível em: <http://www.Irrd.org//rrd21/ 1/heim21005.htm> Acesso em: 23 jan. 2012.

ONAWUMNI, G.O.; YISAK, W.A.; OGUNLANA, E.O. Antibacterial constituents in the essential oil of Cymbopogon citratus (DC.) Stapf. Journal of Ethnopharmacology, v.12, n.3, p.279-86, 1984.

SOARES, M.C.S.C. Avaliação comparativa da eficácia de fitoterápicos e produtos químicos carrapaticidas no controle de Boophilus microplus (Canestrini, 1887) por meio do biocarrapaticidograma. 2003. 98p. Dissertação (Mestrado em Ciências Veterinárias) Departamento de Ciências Veterinárias Universidade Federal Rural de Pernambuco, Recife.

SILVA, W.W. et al. Ação do extrato alcoólico do capim santo (Cymbopogon citratus (DC.) Stapf) sobre nematóides gastrintestinais de ovinos. Agropecuária
Científica no Semi-Árido, v.1, p.46-9, 2005.

SILVA, W.W. et al. Efeitos do neem (Azadirachta indica A. Juss.) e do capim santo [Cymbopogon citratus (DC.) Stapf] sobre os parâmetros reprodutivos de fêmeas ingurgitadas de Boophilus microplus e Rhipicephalus sanguineus (Acari: Ixodidae) no semiárido paraibano. Revista Brasileira de Plantas Medicinais, v.9, n.3, p.1-5, 2007.

SILVA, F.F. et al. Avaliação comparativa da eficácia de fitoterápicos e produtos químicos carrapaticidas no controle do Boophilus microplus (Canestrini, 1887) por meio do biocarrapaticidograma. Medicina Veterinária, v.2, n.3, p.1-8, 2008.

SILVA, M.A.L. et al. Avaliação da composição química de Cymbopogon citratus Stapf cultivado em ambientes com diferentes níveis de poluição e a influência na composição do chá. Universidade Federal de Pernambuco, Acta Scientiarum, Health Sciences, v.32, n.1, p.67-72, 2010. 